\title{
Surgical Results after Unilateral Laminectomy for the Removal of Spinal Cord Tumors
}

\author{
Jung-Heon Lee, Jae-Won Jang, Sung-Hyun Kim, \\ Hyung-Sik Moon, Jung-Kil Lee, Soo-Han Kim \\ Department of Neurosurgery, Chonnam National University School of Medicine \\ \& Hospital Research Institute of Clinical Medicine, Gwangju, Korea
}

Objective: To reduce the risk of postoperative spinal instability or deformity, unilateral laminectomy (UL) has been recommended to remove spinal space-occupying lesions. The purpose of this study was to determine whether there were any advantages of UL for removal of spinal cord tumors.

Methods: From May 1995 to May 2010, 94 patients with spinal cord tumors, who underwent tumor removal via UL in our institute, were enrolled in this study. Intramedullary spinal cord tumors were excluded. Simple radiographs were obtained for accessing the restoration of the spinal column. Spinal magnetic resonance imaging (MRI) was also obtained during the follow-up period to evaluate tumor recurrence.

Results: There were 51 women and 43 men; their mean age was 47.8 years (range, 9-83 years). The mean follow-up period was 52.6 months (range, 24 month-16 years). The sites of the tumors were cervical in 21 cases, thoracic in 37, lumbar in 33, and sacral in 3. These cases included 85 intradural extramedullary (IDEM) and 9 extradural (ED) lesions. IDEM tumors consisted mainly of neurilemmomas (56.3\%) and meningiomas (22.3\%).Tumors were totally removed in 80 cases and subtotally removed in 14 cases. Postoperative neurological status was improved in 53 cases, unchanged in 31 cases, and worsened in 10 cases. During follow-up, MRI showed tumor recurrence in 4 patients. Histopathologically, three cases were meningiomas and one case was neurilemmoma. None of the patients showed spinal instability or kyphotic deformity at last follow-up. Conclusion: UL is an optimal approach for providing sufficient exposure of spinal cord tumors without development of postoperative spinal instability and regional sagittal imbalance in any location of spinal column. In cases of meningioma, careful long-term follow up is needed due to it's relatively high recurrence rate after removal via UL.

Key Words: Unilateral laminectomy $\cdot$ Spinal cord tumors $\cdot$ Surgical result

\section{INTRODUCTION}

Goals in the surgery of the spinal cord tumor are to obtain adequate exposure of the lesion, to remove the lesion totally without injury to the spinal cord and nerve roots, and to preserve spinal column stability. Total laminectomy is the most conventional approach for surgical removal of a spinal cord tumor. It offers a familiar exposure and wide views of the surgical fields to the spinal surgeons. However, many clinical and biomechanical studies have shown that total laminectomy

\footnotetext{
- Received: April 13, 2012 - Revised: September 4, 2012

- Accepted: September 26, 2012

Corresponding Author: Jae-Won Jang, MD

Department of Neurosurgery, Chonnam National University Hospital,

671, Jebongno, Dong-gu, Gwangju 501-757, Korea

Tel: +82-62-220-6600, Fax: +82-62-224-9865

Email: genius60@hanmail.net
}

destroys dorsal bony structures, the interspinous ligament complex, and paraspinal muscles. Therefore, if total laminectomy is done at multiple levels of the spine, it may pose great risk for development of spinal deformity and instability after the surgery. The incidence of spinal deformity after total laminectomy has been reported to be approximately 50\%, especially in children before they reach bone maturity ${ }^{5,29)}$. It also has several disadvantages, such as epidural fibrosis, the absence of osseous protection for the spinal cord, and the development of postoperative severe axial pain.

Unilateral laminectomy (UL) has been recommended to minimize iatrogenic trauma during surgery and the development of postoperative instability or sagittal imbalance. The main advantages of UL is the complete preservation of posterior static structures of the vertebral column, such as the spinous processes, the interspinous and supraspinous ligaments, and the unilateral preservation of the intervertebral joints, laminae, ligamentum flavum and paraspinal muscles. The purpose of this 
study was to determine whether there are advantages of UL for the removal of spinal cord tumors and spinal column restoration during follow-up periord.

\section{MATERIALS AND METHODS}

\section{Patient population}

Since 1995, UL has been used routinely for the removal of all spinal cord tumors with the exception of those intramedullary tumors which generally required total laminectomy and extradural tumors which extensively invaded local soft tissue structures bilaterally. The patient with previous spine surgery was also excluded. The author retrospectively analyzed 94 consecutive patients with spinal cord tumors who underwent tumor removal through a UL between May 1995 and April 2010 in our institute. Information regarding clinical presentation, tumor characteristics, operative findings, postoperative results, and complications were collected from medical and radiological records. Pathological reports on the spinal cord tumor were also reviewed.

\section{Surgical technique}

All patients were placed in a prone position. After the determi nation of the surgical level with intraoperative fluoroscopy, a longitudinal midline incision was done. Paravertebral muscles were detached from the posterior bony structure on the affected side, and laminectomy was done using a high speed drill and punch under a microscope. The base of the spinous process was also drilled until the contralateral roots or dural curve was seen (Fig. 1). The attachments of the contralateral muscles and the interspinous ligaments were kept intact. If required, medial parts of the ipsilateral articular process were removed. The facet joints were not sacrificed unless there was an extra spinal extension of the tumor. Oblique tilting of the operating microscope or operating table to the contralateral side provided an adequate view for extradural and intradural procedures. For intradural lesions, the paramedian incision of the dura was made longitudinally, and the dural margin was tacked to the muscle or fascia laterally. The arachnoid membrane was incised sharply and dissected from the tumor. Tumor removal was performed by internal debulking of the solid tumor or piecemeal resection after meticulous hemostasis and dissection. Tumors with cystic components were easily removed after draining cystic fluid by puncture or aspiration. In cases of intradural spinal meningioma, the site of origin of the tumor was coagulated. Applying cottonoid to the upper and lower pole helped to prevent the spread of blood clots

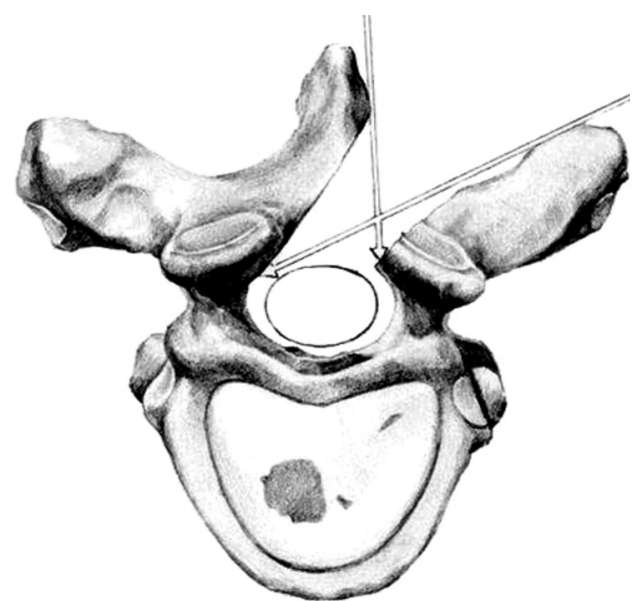

Fig. 1. Schematic view after unilateral hemilaminectomy for exposure of the spinal canal.

into the subarachnoid space. The dura was tightly closed with continuous non-absorbable sutures.

\section{Radiologic assessment}

Simple plain radiographs were obtained before surgery, immediately after the surgery, and at final follow up to evaluate the development of spinal instability or kyphotic deformity. The criteria for spinal instability of cervical spine were anterior displacement more than $3.5 \mathrm{~mm}$, or segmental angle change more than 11 degrees in flexion and extension spinal radiographs. In thoracic or lumbosacral spine, the criterion for spinal instability was anterior displacement more than $2 \mathrm{~mm}$ in flexion and extension spinal radiographs. To evaluate the progression of spinal deformity after UL, C2-C7 angle in cervical spine, T4-T12 angle in thoracic spine, and L1-S1 angle in lumbosacral spine were measured, respectively. Preoperatively, spinal magnetic resonance imaging (MRI) was done to evaluate tumor size and location. During follow-up, MRI was done for evaluation of tumor recurrence.

\section{Statistical analysis}

Data were analyzed using the SPSS program for Windows V17.0 (SPSS, Chicago, IL, U.S.A.); Wilcoxon Signed-Rank test was used for analyses. Data are presented as mean \pm standard deviation. For all analyses, a p-value of $<0.05$ was considered statistically significant.

\section{RESULTS}

We enrolled 51 women and 43 men, their mean age was 46.9 years (range, 9-83 years). Mean follow up duration was 
52.6 months (range, 24 months-16 years). All patients suffered from local or radicular pain and sensory or motor disturbance of the extremities. Preoperative neurological examinations revealed sensory disturbances in 38 patients, weakness of the extremities in 36 patients, and sphincter dysfunction in 9 patients. The sites of the tumors were cervical in 21 cases, thoracic in 37 cases, lumbar in 33 cases and sacral in 3 cases. These cases included 85 intradural extramedullary (IDEM) and 9 extradural (ED) lesions. IDEM tumors consisted mainly of neurilemmomas (56.3\%) and meningiomas (22.3\%). The location and histopathological findings of our cases are shown in Table 1.

In most UL cases, lesions were exposed at two levels (1 level: 19, 2 levels: 51, 3 levels: 23, 4 levels: 1). The operation time ranged from 1 to 5 hours for the removal of spinal cord tumors. Blood transfusion was not necessary during the operation in any of the patients. Tumors were removed gross totally in 80 cases and subtotally in 14 cases according to intraoperative findings (Table 2). In the 14 cases of subtotally removed tumors, it was not done subtotally because of a narrow surgical field, but because of the characteristics of the tumors and the invasiveness of the tumors into neural structures. Postoperative

Table 1. The location and histopathological findings of spinal cord tumors in this study

\begin{tabular}{|c|c|c|c|}
\hline Site & IDEM & ED & Total \\
\hline \multirow[t]{3}{*}{ Cenvical } & Neurilemmoma (13) & Neurilemmoma (1) & 21 \\
\hline & Meningioma (6) & & \\
\hline & Neurofibroma (1) & & \\
\hline \multirow[t]{5}{*}{ Thoracic } & Neurilemmoma (17) & Metastatic cancer (3) & 37 \\
\hline & Meningioma (12) & Fibrous harmatoma (1) & \\
\hline & Metastatic cancer (1) & Lymphoma (1) & \\
\hline & Neural fibrolipoma (1) & & \\
\hline & $\begin{array}{l}\text { Malignant peripheral } \\
\text { nerve sheath tumor (1) }\end{array}$ & & \\
\hline \multirow[t]{10}{*}{ Lumbar } & Neurilemmoma (20) & Burkitt's lymphoma (1) & 33 \\
\hline & Meningioma (3) & & \\
\hline & Teratoma (2) & & \\
\hline & Ependymoma (1) & & \\
\hline & Lipoma (1) & & \\
\hline & Epidermoid cyst (1) & & \\
\hline & PNET (1) & & \\
\hline & Myxchondroma (1) & & \\
\hline & Neurofibroma (1) & & \\
\hline & Metastatic cancer (1) & & \\
\hline \multirow[t]{2}{*}{ Sacral } & Neurilemmoma (1) & Neurilemmoma (1) & 3 \\
\hline & & Chondrosarcoma (1) & \\
\hline Total & 85 & 9 & 94 \\
\hline
\end{tabular}

neurological status was improved in 53 cases, unchanged in 31 cases, and worsened in 10 cases. During the follow-up period, neurologic symptoms were gradually improved in 7 and of 10 worsened patients, but were not changed in 3 patients. Most patients whose neurological status remained unchanged or worsened had a long symptom duration before the surgery. Postoperative complications included one epidural hematoma, one wound infection, and one cerebrospinal fluid (CSF) leakage. The epidural hematoma and wound infection were resolved after revision and hematoma removal, and debridement and antibiotic treatment, respectively. CSF leakage was managed by lumbar puncture drainage. All three patients had a favorable outcome.

There were 4 patients who underwent an operation due to a recurrent tumor. For all of them, tumors were gross totally removed at the time of the initial operation. Histopathologically, three cases were meningiomas in the thoracic spine, and they had developed at the same thoracic spinal level but on the contralateral side. One recurring case was a neurilemmoma in the lumbar spine. In our institution, the recurrence rate of spinal meningioma was $12.5 \%$ (3/24). The mean recurrence period was 12.3 years after the first surgery. In cases of neurilemmoma, recurrence occurred in 1 case (1.8\%) at 10.1 years after the surgery.

None of the patients developed spinal instability on simple plain radiographs during follow-up. In cases with cervical spinal

Table 2. The extents of the tumor resection according to pathologic findings

\begin{tabular}{lcc}
\hline \hline & $\begin{array}{c}\text { Growth Total } \\
\text { Removal }\end{array}$ & $\begin{array}{c}\text { Subtotal } \\
\text { Removal }\end{array}$ \\
\hline Neurilemmoma & 49 & 4 \\
Meningioma & 20 & 2 \\
Chondrosarcoma & 1 & \\
Metastatic tumor & 3 & 1 \\
Ependymoma & 1 & \\
Epidermoid cyst & 1 & \\
Lipoma & 1 & \\
Malignant lymphoma & 1 & \\
Myxochondroma & 1 & \\
Burkitt lymphoma & 1 & \\
PNET & & 1 \\
Fibroadipose harmatoma & & 1 \\
Mature cystic teratoma & & 1 \\
Mature teratoma & & 1 \\
Neurofibroma & 1 & 1 \\
Suspicious of neural fibrolipoma & & 1 \\
Malignant peripheral nerve sheath tumor & & 1 \\
\hline
\end{tabular}


Table 3. The changes of regional sagittal angles after hemilaminectomy ( $p>0.05$, Wilcoxon Signed-Rank Test)

\begin{tabular}{lccc}
\hline \hline & Cenvical (21 cases) & Thoracic (37 cases) & Lumbosacral (36 cases) \\
\hline Preoperative angle & $-22.8 \pm 10.3$ & $19.8 \pm 7.5$ & $-30.5 \pm 7.2$ \\
Last follow-up angle & $-20.5 \pm 11.3$ & $17.2 \pm 6.4$ & $-33.8 \pm 9.2$ \\
p-value & $>0.05$ & $>0.05$ & $>0.05$ \\
\hline
\end{tabular}

Values are means $\pm S D$

cord tumors, the average $\mathrm{C} 2-\mathrm{C} 7$ angles in preoperative and last follow-up radiographs were $-22.8 \pm 10.3$ and $-20.5 \pm 11.3$ respectively. In cases with thoracic spinal cord tumors, the average T4-T12 angles in preoperative and last follow-up radiographs were $19.8 \pm 7.5$ and $17.2 \pm 6.4$ respectively. The average L1-S1 angles in preoperative and last follow-up radiographs were $-30.5 \pm 7.2$ and $-33.8 \pm 9.2$ respectively in cases of lumbar spinal cord tumors. Significant differences were not found between preoperative and last follow-up C2-C7 angles, T4-T12 angles, and L1-S1 angles ( $\mathrm{p}>0.05)$ (Table 3).

\section{Illustrative Case}

\section{Case 1}

An 28-year-old man suffered from neck pain and motor weakness of the right arm (grade 3/5). Preoperative MRI showed a large enhancing IDEM dumbbell shaped mass extending from $\mathrm{C} 2$ to $\mathrm{C} 3$ involving the right side of the spinal canal and C2-3 neural foramen. The spinal cord was compressed and displaced left-posteriorly by the mass (Fig. 2A and B). C2-3 right UL was performed, and the tumor was totally removed. The postoperative course was uneventful, with gradual improvement of the patient's neurological function. The histopathological findings were consistent with the those of a schwannoma. Postoperative enhanced computed tomography (CT) revealed complete removal of the spinal cord tumor (Fig. 2C). A plain radiograph at two year follow-up demonstrated no evidence of spinal instability (Fig. 2D).

\section{Case 2}

A 47-year-old woman presented with paraparesis of both legs (grade 4/5) and decreased sensation below the T8 sensory dermatome. A thoracic MRI revealed a well demarcated IDEM tumor with dural tail sign in the T7 spinal canal. Strong homogenous enhancement was appreciated after gadolinium administration (Fig. 3A and B). With a T6-7-8 UL on the right side, the tumor was removed gross totally. Broad-based dural attachment was coagulated by a bipolar, and the dural origin of the tumor was not excised. The postoperative course was uneventful, with notable improvement in neurological function. The histopathology revealed a diagnosis of meningioma.

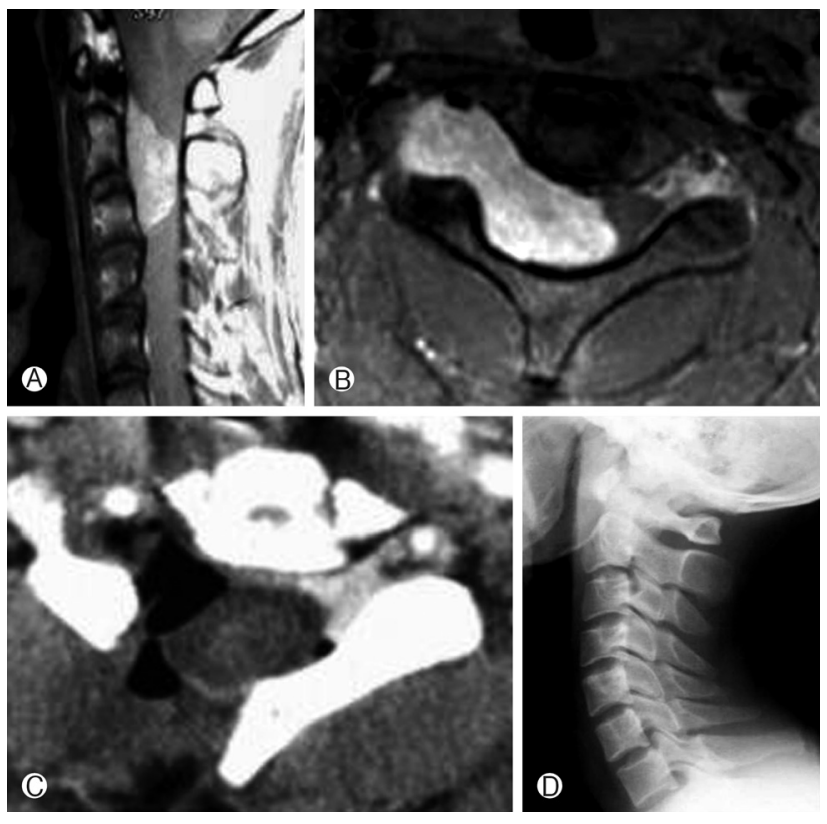

Fig. 2. Case illustration 1. (A and B) Preoperative gadolinium enhanced magnetic resonance imaging (MRI) revealed a large enhancing intradural extramedullary dumbbell shape mass extending from C2 to $\mathrm{C} 3$ and compression of the spinal cord. (C) Postoperative computed tomography (CT) scan showed complete tumor removal via a right unilateral hemilaminectomy. (D) A plain radiograph at two year follow-up (D) demonstrates no evidence of spinal instability.

Paraparesis reappeared 7 years after the initial surgery. MRI demonstrated a recurrent meningioma on the contralateral side to the previous operation site (Fig. 3C and D). A T7-8 total laminectomy was done by using a drill, curettes, and punches. After a midline longitudinal dural incision, gross total tumor removal was done. After the surgery, paraparesis was gradually improved to Grade (IV/IV+) with rehabilitation treatment.

\section{DISCUSSION}

Although extensive laminectomy has been widely used for the removal of spinal cord tumors, it also has associated postoperative complications. The integrity of ligament flavum and of supraspinous, and interspinous ligaments is known to be crucial for the dynamic stability of the spine. Spinal instability 

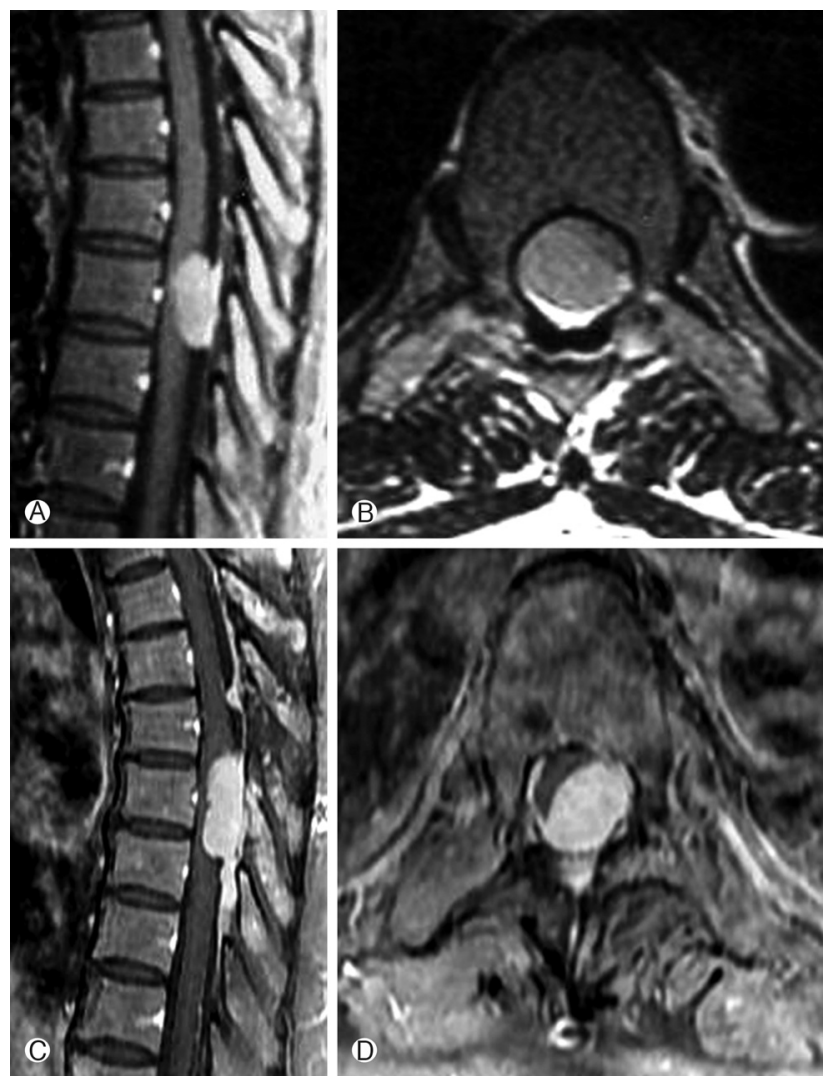

Fig. 3. Case illustration 2. (A and B) Preoperative gadolinium enhanced MRI revealed a large enhancing intradural extramedullary mass with broad dural enhancement on T7. (C and D) At thirteenyear follow-up, MRI axial (C) and sagittal (D) images showed a recurrent tumor at the previous operation site. However, the tumor origin site was on the contralateral side.

and deformity can occur due to damage to the posterior bony structure, interspinous ligament complex, and paraspinal muscles $^{2,5,10-12,28-30)}$. The development of a kyphotic deformity has been frequently reported after total laminectomy for spinal disease. It results in an anterior compression of the spinal cord and progressive myelopathy secondarily. To reduce the risk for development of spinal instability or deformity after laminectomy, less invasive techniques, such as UL, laminoplasty, or osteoplastic laminotomy, have been applied for the removal of spinal space occupying lesions ${ }^{4,11,20)}$. Some authors have advocated that laminoplasty has advantages of maintaining postoperative stability and preventing epidural scar formation. Inoue et al. ${ }^{12)}$ reported that upper cervical spine kyphosis and compensatory increasing lordosis of the lower cervical spine were frequently observed in the laminectomy group compared with the laminoplasty group. However, laminoplasty also disrupts the posterior ligamentous structures. Moreover, laminoplasty is difficult to apply in the thoracic and lumbar spine because the pedicles have a tendency to fracture due to their anatomical structure. Another surgical alternative is osteoplastic laminotomy, in which the removed bone elements are repositioned at the end of the operation ${ }^{18,20)}$. However, this technique is time consuming, and the destruction of the posterior supporting structure is unavoidable. By contrast, UL induces less injury to the dynamic dorsal structures of the vertebral column including ligamentous structures and paraspinal muscles, compared with total laminectomy, laminoplasty, or osteoplastic laminotomy.

UL has been widely applied to spinal surgery, especially degenerative spine disease ${ }^{13,15,26}$. The technique avoids damage to the supraspinous and interspinous ligaments and the paravertebral muscles of the opposite side, and is superior to laminoplasty in the maintenance of both the alignment and the mobility by preserving more paravertebral structures. In addition, UL can provide an adequate surgical window with development of microsurgical techniques ${ }^{1-4,6-8,17,19,21,22,25)}$. Mariconda et al. ${ }^{15)}$ reported that there was not any evidence of instability among the people who underwent bilateral decompression of lumbar spinal stenosis using unilateral laminectomy. Several authors recommended UL for various spinal cord tumors ${ }^{7,827)}$. Takashi et al. ${ }^{2)}$ advocated that worsening of the spinal curvature was significantly less frequent in the UL group than in the conventional laminectomy or the laminoplasty group. In this study, we evaluated about the development of spinal deformity and instability. In cervical and lumbar spine, overall alignment (C2-C7 and L1-S1) was measured. Followup radiologic results showed the good maintenance of cervical and lumbar lordosis compared to preoperative radiologic results. In thoracic spine, we measured the angle of T4-T12 angle, and it showed a similar result compared with preoperative data. Moreover, the development of spinal instability in cervical, thoracic, and lumbar spine was not observed during followup. These facts indicate that UL can maintain spinal stability and alignment after the surgery by avoiding damage to the ligamentous structures, intervertebral joints, and paravertebral spinal muscles of the opposite side. In this view, we encourage early ambulation after postoperative day 2 , and patients can avoid external bracing and reduce hospital stays. In addition, less epidural scarring is expected after unilateral removal of ligamentum flavum and osseous protection for the spinal cord by remaining bony structures.

Some surgeons may be concerned about incomplete removal of the tumor in UL, or inadvertent spinal cord damage with the relatively narrow surgical corridor compared with total conventional laminectomy during the surgery for spinal cord tumors. However, the narrow surgical field in UL is not an obstacle to removing IDEM and ED tumors. Under the microscope, we secured working space for the operation via drilling of the base of the spinous process. We then, could gross totally 
remove the tumor mass in the majority of cases. The exposure of the dorsal parts of the spinal cord after creating the working space for the operation is sufficient in comparison to total laminectomy. The dissection of the arachnoidal compartments and the release of cerebrospinal fluid provided a larger field for surgical manipulation. When gross total removal of the tumor without cord damage is not feasible through the unilateral procedure, the surgeon can convert to bilateral laminectomy during the operation. In this study, there were 14 cases of subtotal removal of tumor. However, none were because of a narrow surgical field; rather, it was because of the characteristics of and the invasiveness of the tumors. Ten cases of clinically worsening after surgery also achieved sufficient surgical field via UL and microscopic view. Among them, seven cases were subtotally removed cases due to adherence with adjacent spinal cord. Therefore, spinal cord might be injured due to retraction or thermal force. Future, the analysis about the worsening of neurological symptom will be required with large number cases. We experienced recurrent tumors in four patients after the surgery. Histopathologically, these cases were spinal meningioma in the thoracic spine, and the recurrent tumors occurred on the contralateral side at the same spinal level, except for one case which was diagnosed as a neurilemmoma. Although the four recurrent tumors had initially been removed gross totally, tumors redeveloped during follow-up.

Schick et al. ${ }^{23)}$ reported surgical results of 197 benign spinal neoplasms; they showed that $79.7 \%$ were completely removed and the recurrence rate was $10.2 \%$ over an average of 4.3 years postoperatively. The overall recurrence rate was 4.8 $\%$ in the present study, which is similar or superior compared to other clinical studies ${ }^{7,23,27}$. King et al. ${ }^{14)}$ reported one recurrence of spinal meningioma in 78 patients 14 years after the first operation, and the tumor recurred in one case that had been totally resected in the first operation. However, the dural bases were diathermied and not excised because of the anterior placement. Mirimanoff et al. ${ }^{16)}$ found a recurrence incidence of $13 \%$ over 10 years in spinal meningioma, and recurrence of spinal meningiomas was reported as being delayed up to 38 years ${ }^{9)}$. In our series, the recurrence rate in spinal meningioma was $12.5 \%$ with a mean recurrence period of 12.3 years. In recurrent cases of meningioma, the tumors crossed the midline of the spinal canal on pre-operative MRI. The tumors had been gross totally removed via UL using a microscopic view in the first operation. The site of dural attachments to the tumor was coagulated to avoid additional risks of CSF leakage after removing of dura and spinal cord damage related to dural excision of the tumor origin site. Follow-up MRI in all patients who had recurrent meningioma revealed that tumors dominantly sited at contralateral spinal canal. Therefore, we can't exclude the possibility that the du- ral coagulation may influence lowering of the tumor recurrence. However, unlike intracranial meningiomas, removing the dural origin seems to make little difference to recurrence rates in literature reviews ${ }^{23,24)}$. In our cases of recurred meningioma, all tumors were dominant at the contralateral side of laminectomy, not at the previous operation site, which indicates that diathermy of the dural origin may be efficient for reducing recurrence of spinal meningioma. Although tumor recurrence was higher in spinal meningioma compared to other spinal tumors, spinal meningioma can be successfully resected and coagulated at its dural origin via UL. To reduce the recurrence rates of spinal meningioma, it will be important to estimate the extent of dural origin through careful observation of preoperative radiologic studies, especially, whether the origin site spreads the contralateral area of the surgical approach or not. If the origin site of tumor is spread to the contralateral side of approach, diathermy of the dural attachment will be important. Lastly, even if gross total removal of tumor was done, careful long-term follow up is essential due to delayed recurrence, especially in cases of meningioma.

\section{CONCLUSION}

Unilateral laminectomy for removal of spinal cord tumors is an optimal approach that can yield excellent exposure of the spinal canal and gross total removal of the lesion without any postoperative development of spinal instability or deformity. Furthermore, after this procedure, our patients are encouraged to achieve early mobilization and hospital stays may be reduced because the stability of spinal column after UL has been well maintained during follow-up. In cases of spinal meningioma, evaluation of the origin site via preoperative radiologic studies and diathermy of the dural attachments will be important to reduce tumor recurrence.

\section{Acknowledgements}

This study was supported by a grant (CRI12027-1) Chonnam national university hospital research institute of clinical medicine.

\section{REFERENCES}

1. Agrawal A, Cincu R, Wani B: [Modified posterior unilateral laminectomy for a complex dumbbell schwannoma of the thoracolumbar junction]. Acta Orthop Traumatol Turc 43:535-539, 2009

2. Asazuma T, Nakamura M, Matsumoto M, Chibo K, Toyama Y: Postoperative changes of spinal curvature and range of motion 
in adult patients with cervical spinal cord tumors: analysis of 51 cases and review of the literature. J Spinal Disord Tech 17:178-182, 2004

3. Balak $\mathrm{N}$ : Unilateral partial hemilaminectomy in the removal of a large spinal ependymoma. Spine J 8:1030-1036, 2008

4. Bertalanffy $\mathrm{H}$, Mitani S, Otani M, Ichikizaki K, Toya S: Usefulness of hemilaminectomy for microsurgical management of intraspinal lesions. Keio J Med 41:76-79, 1992

5. Cattell HS, Clark GL Jr: Cervical kyphosis and instability following multiple laminectomies in children. J Bone Joint Surg Am 49:713-720, 1967

6. Cha JW, Lee JS, Ha HG: Posterior hemilaminectomy approach to anterolaterally located intradural extramedullary spinal cord tumors. Korean J Spine 1:500-506, 2004

7. Chiou SM, Eggert HR, Laborde G, Seeger W: Microsurgical unilateral approaches for spinal tumour surgery: eight years' experience in 256 primary operated patients. Acta Neurochir (Wien) 100:127-133, 1989

8. Eggert HR, Scheremet R, Seeger W, Gaitzsch J: Unilateral microsurgical approaches to extramedullary spinal tumours. Operative technique and results. Acta Neurochir (Wien) 67:245-253, 1983

9. Feiring EH, Barron K: Late recurrence of spinal-cord meningioma. J Neurosurg 19:652-656, 1962

10. Herman JM, Sonntag VK: Cervical corpectomy and plate fixation for postlaminectomy kyphosis. J Neurosurg 80:963-970, 1994

11. Hong-Wan N, Ee-Chon T, Qing-Hang Z: Biomechanical effects of C2-C7 intersegmental stability due to laminectomy with unilateral and bilateral facetectomy. Spine (Phila Pa 1976) 29: 1737-1745, 2004

12. Inoue A, Ikata T, Katoh S: Spinal deformity following surgery for spinal cord tumors and tumorous lesions: analysis based on an assessment of the spinal functional curve. Spinal Cord 34: 536-542, 1996

13. Kim JS, Jung B, Arbatti N, Lee SH. Surgical experience of unilateral laminectomy for bilateral decompression (ULBD) of ossified ligamentum flavum in the thoracic spine. Minim Invasive Neurosurg 52:74-78, 2009

14. King AT, Sharr MM, Gullan RW, Bartlett JR. Spinal meningiomas: a 20-year review. Br J Neurosurg 12:521-526, 1998

15. Mariconda M, Fava R, Gatto A, Longo C, Milano C: Unilateral laminectomy for bilateral decompression of lumbar spinal stenosis: a prospective comparative study with conservatively treated patients. J Spinal Disord Tech 15:39-46, 2002

16. Mirimanoff RO, Dosoretz DE, Linggood RM, Ojemann RG, Martuza RL: Meningioma: analysis of recurrence and progre- ssion following neurosurgical resection. J Neurosurg 62:18-24, 1985

17. Oktem IS, Akdemir H, Kurtsoy A, Koç RK, Menkü A, Tucer B: Hemilaminectomy for the removal of the spinal lesions. Spinal Cord 38:92-96, 2000

18. Parkinson D. Replacement laminotomy: Surg Neurol 8:277279, 1977

19. Pompili A, Caroli F, Cattani F, Crecco M, Giovannetti M, Raus L, et al: Unilateral limited laminectomy as the approach of choice for the removal of thoracolumbar neurofibromas. Spine (Phila Pa 1976) 29:1698-1702, 2004

20. Raimondi AJ, Gutierrez FA, Di Rocco C: Laminotomy and total reconstruction of the posterior spinal arch for spinal canal surgery in childhood. J Neurosurg 45:555-560, 1976

21. Reimer R, Onofrio BM: Astrocytomas of the spinal cord in children and adolescents. J Neurosurg 63:669-675, 1985

22. Sario-glu AC, Hanci M, Bozkus H, Kaynar MY, Kafadar A: Unilateral hemilaminectomy for the removal of the spinal spaceoccupying lesions. Minim Invasive Neurosurg 40:74-77, 1997

23. Schick U, Marquardt G, Lorenz R: Recurrence of benign spinal neoplasms. Neurosurg Rev 24:20-25, 2001

24. Solero CL, Fornari M, Giombini S, Lasio G, Oliveri G, Cimino C, et al: Spinal meningiomas: review of 174 operated cases. Neurosurgery 25:153-160, 1989

25. Sridhar K, Ramamurthi R, Vasudevan MC, Ramamurthi B: Limited unilateral approach for extramedullary spinal tumours. Br J Neurosurg 12:430-433, 1998

26. Thome C, Zevgaridis D, Leheta O, B?zner H, P?ckler-Sch?niger $\mathrm{C}$, W?hrle J, et al: Outcome after less-invasive decompression of lumbar spinal stenosis: a randomized comparison of unilateral laminotomy, bilateral laminotomy, and laminectomy. J Neurosurg Spine 3:129-141, 2005

27. Yasargil MG, Tranmer BI, Adamson TE, Roth P: Unilateral partial hemi-laminectomy for the removal of extra- and intramedullary tumours and AVMs. Adv Tech Stand Neurosurg 18: 113-132, 1991

28. Yasuoka S, Peterson HA, Laws ER Jr, MacCarty CS: Pathogenesis and prophylaxis of postlaminectomy deformity of the spine after multiple level laminectomy: difference between children and adults. Neurosurgery 9:145-152, 1981

29. Yasuoka S, Peterson HA, MacCarty CS: Incidence of spinal column deformity after multilevel laminectomy in children and adults. J Neurosurg 57:441-445, 1982

30. Zander T, Rohlmann A, Klockner C, Bergmann G: Influence of graded facetectomy and laminectomy on spinal biomechanics. Eur Spine J 12:427-434, 2003 